\title{
The Role of Glycogen in Biological Cycle of Trichinella spiralis
}

\author{
Evgenya A. Sidor and Oleg N. Andreyanov* \\ All-Russian Scientific Research Institute of Fundamental and Applied Parasitology of Animals and Plants named after K.I. Skryabin - \\ Branch of the Federal State Budget Scientific Institution «Federal Scientific Center - All-Russian Scientific Research Institute of \\ Experimental Veterenari Medicina K.I. Skryabin and Y.R. Kovalenko, the Russian Academy of Sciences», B. Cheremushkinskaya \\ Street 28, 117218 Moscow, Russia.
}

*Corresponding author's Email: 1980oleg@mail.ru; (DORCID: 0000-0003-3357-9322

\begin{abstract}
The energy sources of Trichinella spiralis change in both the muscular and intestinal stages of its life in the host organism. The purpose of this study was to investigate the quantitative changes in glycogen concentration during the life cycle of $T$. spiralis in a host organism. Trichinella spiralis was passaged on laboratory rodents under the vivarium conditions. Sixty-nine white rats $(350 \mathrm{~g}$ each) were infected with $T$. spiralis at a dose of 5 muscle larvae/gram of body weight. The animals were euthanized at different time periods from the start of the experiment. Trichinella muscle larvae were isolated by artificial fermenting meat mince in gastric juice. To determine the viability of Trichinella larvae, they were heated to $38 \pm 2{ }^{\circ} \mathrm{C}$ for 10 minutes their motor activity was investigated. $\left(38 \pm 2{ }^{\circ} \mathrm{C}\right)$. To determine the invasive properties of $T$. spiralis at different stages of its development in rats, the muscular larvae isolated from the rat muscles were used to infect laboratory mice. The invasive capacity of $T$. spiralis was assessed on day 45 post-infection. For the study of intestinal Trichinella larvae, laboratory rats were not fed a day before infection. Adult nematodes were isolated from the small intestine of laboratory rats at 3, 6 and 24 hours post-infection. The nematodes were counted in the Migacheva-Kotelnikov chamber in each individual sample. The concentration of glycogen in the nematodes was calculated according to the quantitative method for determining glycogen in Trichinella larvae. Low glycogen levels in the muscle larvae were observed on day 14 post-infection. The glycogen concentration in muscular larva was $0.0054 \pm 0.0027 \mu \mathrm{g} /$ larva on day $21,0.0136 \pm 0.0024 \mu \mathrm{g} / \mathrm{larva}$ on day 28 , and $0.0771 \pm 0.0025 \mu \mathrm{g} /$ larva on day 45 after the rats were infected. Maximum concentration of glycogen was recorded 4 months post-infection $(0.0930 \pm 0.0029 \mu \mathrm{g} / \mathrm{larva})$. Further, the glycogen level began to decrease slowly. In the 20th month post-infection, after infection, the amount of glycogen in a Trichinella larva was $0.0786 \pm 0.0023 \mu \mathrm{g}$. In the body of intestinal nematodes, 3 hours after infecting the animals, the glycogen concentration was reduced to $0.0472 \pm 0.0003 \mu \mathrm{g}$ in one nematode. The same time period later, it reached to value of $0.0272 \pm 0.0002 \mu \mathrm{g}$. In intestinal T. spiralis, which remained in the small intestine of rats for 24 hours, the glycogen was not detected. The amount of glycogen at the muscle stage of $T$. spiralis development was extremely important in the first hours of the helminth's residing in the host's intestines. Energy requirements during the period when the helminth cannot obtain enough food depend on the glycogen content. When the glycogen concentration in the parasite is insufficient, the Trichinella larvae will lose their invasion capacity.
\end{abstract}

Key words: Bioassay test; Glycogen; Nematode; Parasitic helminth, Trichinella spiralis

\section{INTRODUCTION}

Glycogen is known to be the main reserve polysaccharide and a key source of energy for helminths. A complex life cycle with a change of environment forces helminths to accumulate a significant amount of reserve substances at different stages of their development. One of these helminths is Trichinella spiralis causing trichinosis, a dangerous disease affecting animals and humans. The muscle larvae of Trichinella spiralis are extremely rich in glycogen, which constitutes 16\% of their dry weight (Beckett and Boothroyd, 1962; Castro and Fairbairn, 1969). Many researchers used the methods of histochemical staining and electron microscopy to find the main locations of glycogen deposition at different stages of Trichinella biological cycle (De Nollin and Van den Bossche, 1973; Ferguson and Castro, 1973, Andreyanov et al, 2019, Rudneva et al., 2019; Sidor and Andreyanov, 2019). It was established that the total amount of glycogen increases during the maturation of the larva in muscle tissue, and then sharply decreases when larvae grow, molt and reach puberty in the intestine of the new host. In the first hours after invasion, intestinal Trichinella relies mainly on endogenous glycogen stores accumulated at the previous stage of development (Ferguson and Castro, 1973; Kilgore et al., 1986). Previous research established that intestinal Trichinella does not survive for over 12 hours in vitro in a cultured medium without nutrients, while muscle larvae can survive for several days (Kozar et al., 1966).

The goal of this study was to obtain data on the glycogen content in Trichinella spiralis, in order to understand the invasiveness level of the causative agent of trichinellosis. 


\section{Ethical approval}

The present experimental research was conducted in compliance with health protection guidelines of experimental animals (European Convention for the Protection of Vertebrate Animals used for Experimental and other Scientific Purposes, ETS No.123. Strasbourg, 18/03/1986.).

\section{Animals and study design}

The experiment was conducted in the All-Russian Scientific Research Institute of Fundamental and Applied Parasitology of Animals and Plants named after K.I. Skryabin, Russia. In this study, 69 white male laboratory rats weighing $350 \mathrm{~g}$ each were used. Larvae of Trichinella spiralis were isolated from the Large White pig and passaged on laboratory rodents (white mice and Wistar rats) in the institute vivarium. The muscle larvae were released from the infected pig muscles by digestion of the pig carcass with $1 \%$ pepsin $(1: 1,000)$ and $1 \%$ hydrochloric acid (Bessonov, 1975). The Wistar rats were infected at a dose of 5 larvae/g of body weight. The rats were euthanized by cervical dislocation at specific time periods, starting on day 14 after infection and ending in month 20. Trichinella muscle larvae were isolated through passive peptolysis (Bessonov, 1975). The viability of Trichinella larvae was determined by their motor activity when they were heated to $38 \pm 2{ }^{\circ} \mathrm{C}$ for 10 minutes (Skvortsova and Uspensky, 2016). In order to identify the invasive properties of the Trichinella larvae, they were collected at different time periods from the infected rats and used to infect laboratory mice. On the $45^{\text {th }}$ day post-infection, the invasive capacity of Trichinella larvae was assessed based on the presence of encapsulated Trichinella larvae in the muscular tissue of the mice (Bessonov, 1975). Adult nematodes were isolated by dissecting the small bowel of the euthanized rats. The dissected and isolated part of the animal's intestinal tract was placed on the surface of a nylon sieve with $0.8 \mathrm{~mm}$ mesh size in the Baermann apparatus at $38 \pm 2^{\circ} \mathrm{C}$ for 6 hours. The sediment with nematodes was then rinsed 5-7 times in the physiological saline. To study adult Trichinella spiralis located in the small intestine of laboratory rats, the rats had not received any food for one day prior to infection. The nematodes were isolated from the small intestine of fasted rats at 3, 6, and 24 hours after the invasion.

\section{Glycogen concentration measurement}

Adult nematodes and muscular larvae of Trichinella spiralis in a sample (one drop of the obtained suspension) were counted using Migacheva-Kotelnikov chamber. The glycogen concentration was calculated according to the method described by Andreyanov et al. (2019) that is based on the treatment of glycogen molecules isolated from Trichinella with iodine reagent prepared according to the method described by Danchenko and Chirkin (2010). Briefly, $0.9 \mathrm{ml}$ of 33\% potassium hydroxide solution was added to the isolated Trichinella (1000-10,000 Trichinella in one drop of the suspended sedimentation). The solution was heated for 20 minutes at $100 \pm 2^{\circ} \mathrm{C}$ and cooled in cold water to $8 \pm 2$ ${ }^{\circ} \mathrm{C}$, then mixed with $1.3 \mathrm{ml}$ of $96 \%$ ethyl alcohol and heated for 5 minutes at $100 \pm 2{ }^{\circ} \mathrm{C}$. The resulting solution was cooled for 5 minutes to $8 \pm 2{ }^{\circ} \mathrm{C}$, then centrifuged for 15 minutes at $3,000 \mathrm{~g}$ and the supernatant was drained. The resulting precipitate was dissolved in $0.2 \mathrm{ml}$ of saturated ammonium chloride solution and reheated for 5 minutes at 100 $\pm 2^{\circ} \mathrm{C}$. The tubes containing the precipitate were cooled to $8 \pm 2{ }^{\circ} \mathrm{C}$ and $0.2 \mathrm{ml}$ of distilled water and $2.6 \mathrm{ml}$ of the iodine reagent were added. In the control sample, $0.2 \mathrm{ml}$ of saturated ammonium chloride solution, $2.6 \mathrm{ml}$ of iodine reagent and $0.2 \mathrm{ml}$ of distilled water were mixed simultaneously. In the resulting solutions, optical density was measured by photoelectric microcalorimeter (МКМФ-02 refractometer) with an optical path length of $5 \mathrm{~mm}$ at a wavelength of 425 $\mathrm{nm}$. In order to measure the glycogen concentration in the samples, a calibration curve was made (the $\mathrm{x}$-axis showing the glycogen content in a sample (mg); the y-axis showing the optical density values of calibration solutions). The slope tangent was 1.3. In order to determine the calibration curve, calibration glycogen solutions containing 0.01 to $0.4 \mathrm{mg}$ of glycogen in a sample were prepared.

The glycogen concentration was calculated according to the following formula 1: $\mathrm{C}=\mathrm{E} \times \mathrm{k} / \mathrm{F}$

Where $\mathrm{C}$ is glycogen concentration ( $\mathrm{mg}$ per 1 drop), $\mathrm{E}$ is the optical density of the analyzed sample, $\mathrm{F}$ is the factor, which is calculated as an obliquity tangent of the calibration curve, $\mathrm{k}$ is dilution coefficient of a sample.

\section{RESULTS AND DISCUSSION}

\section{Glycogen level of Trichinella muscle larvae}

The glycogen concentration was determined at different stages of isolated muscle larvae in the muscle tissue of rats. On the 14th day after the infection, Trichinella muscle larvae showed a low glycogen level of $0.0028 \pm 0.0012$ $\mu \mathrm{g} / \mathrm{larva}$. Larvae of this age are motile, but not invasive. Glycogen level on day 21, 28, and 45 post-infection were $0.0054 \pm 0.0027,0.0136 \pm 0.0024 \mu \mathrm{g} / \mathrm{larva}$, and $0.0771 \pm 0.0025 \mu \mathrm{g} / \mathrm{larva}$, respectively. Maximum glycogen concentration was recorded in 4 months after infection $(0.0930 \pm 0.0029 \mu \mathrm{g} / \mathrm{larva})$. Following that, the glycogen level 
began to decrease slowly $(0.0916 \pm 0.0031 \mu \mathrm{g} / \mathrm{larva}$ at 6.5 month post-infection, $0.0844 \pm 0.0027 \mu \mathrm{g} / \mathrm{larva}$ at $11 \mathrm{month}$ post-infection, and $0.0786 \pm 0.0023 \mu \mathrm{g} /$ larva at 20 month post-infection; Figure 1).

Starting from the 21 st day after the infection, the larvae were invasive, which was confirmed by the results of the bioassay test on laboratory mice. The data on the invasive capacity of the newborn Trichinella muscle larvae complies with the findings of Skvortsova and Uspensky (2018), who showed that the newborn larvae (15-, 16- and 17-day-old) are not invasive. By 20 month post-infection, the number of dead Trichinella larvae was 5\% of total larvae isolated after digestion in artificial gastric juice. Starting from the 11th month after the invasion, lime salts are formed at the poles of the capsule in the muscle tissue.

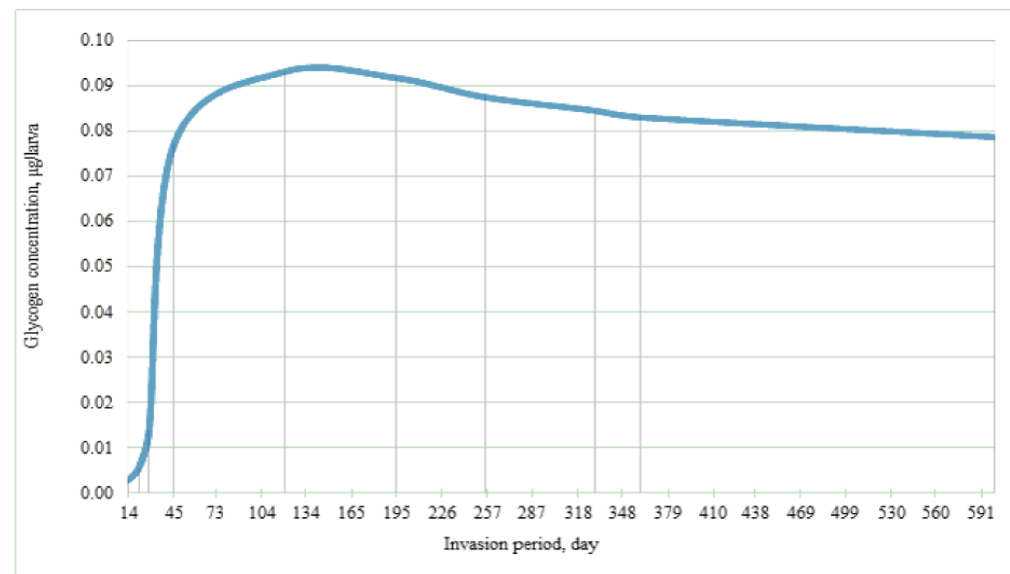

Figure 1. The content of glycogen in muscle larvae of Trichinella spiralis in experimentally infected rats

\section{Adult nematodes}

The rats were infected with muscle larvae that, upon reaching the intestinal tract, begin to grow, molt, differentiate into males and females and turn into adult Trichinella spiralis. Three hours after the infection of the fasted rats, the glycogen concentration in Trichinella spiralis helminths decreased to $0.0472 \pm 0.0003 \mu \mathrm{g} / \mathrm{nematode}$. After the same time period, the glycogen concentration reached to value of $0.0272 \pm 0.0002 \mu \mathrm{g} /$ nematode. In intestinal adult nematodes, which remained in the small intestine of fasted rats for 24 hours, glycogen was not detected (Figure 2).

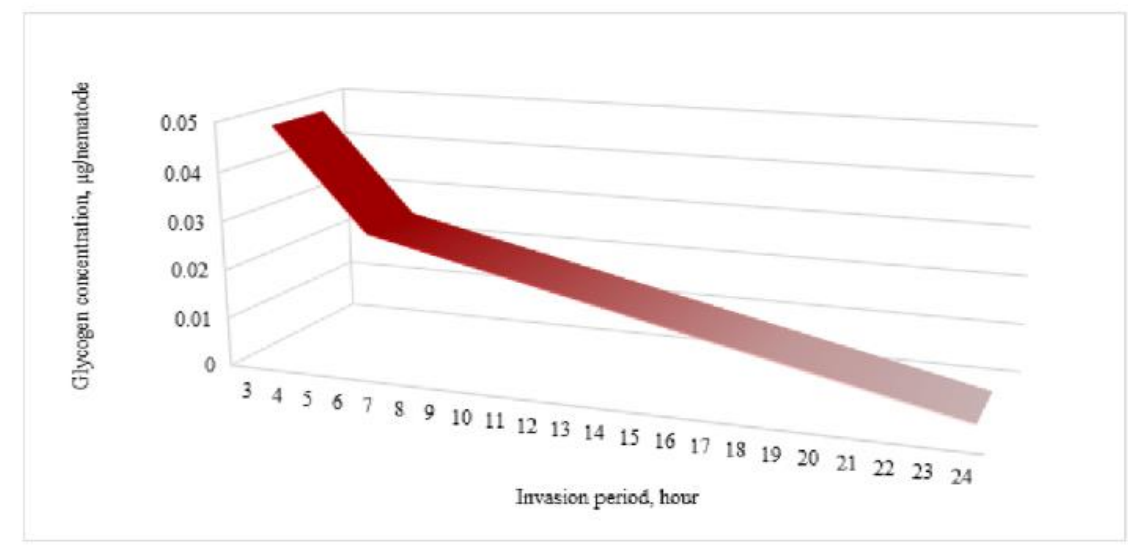

Figure 2. The content of glycogen in intestinal Trichinella spiralis in experimentally infected rats

\section{Invasive capacity of Trichinella muscle larvae}

The invasion of the larvae into the muscle fibers is marked by their intensive organogenesis and growth. Starting from the 7th day of the invasion, the larvae cause enhanced glycolysis of nearby cells and start to accumulate glucose and synthesize glycogen (Stewart, 1976; Montgomery et al., 2003; Okada et al., 2013). The Trichinella larvae become invasive on the 16-17th day after infection, when most larvae finish their organogenesis. Most of the Trichinella muscle larvae become invasive by the $19^{\text {th }}-20^{\text {th }}$ day after the infection. Throughout this experiment, the invasiveness of the larvae remained high, starting from the 21 st day after the infection.

Wu et al. (2009) established that blood glucose levels of invasive mice in the period between the 4th and 28th days after invasion are lower than those of the non-infected ones. An increase in the glycogen content in the affected muscles was also recorded between the 8th and 24th days after infection. The researchers stated that during hypoglycemia and an increase in glycogen levels in the animals' invaded muscles, the storage of reserve polysaccharide in the larvae increase 
rapidly between the 14th and 28th days after the infection (Pereverzeva, 1966). Pereverzeva (1966) and Stewar (1976) also noted a significant increase in glycogen, by more than two times, during this period. It is noteworthy that Kilgore (1986) found that in newborn larvae of Trichinella, glycogen content constituted $7.8 \%$ of their dry weight, whereas, in 45-day-old muscle larvae, glycogen content was $16.1 \%$ (Castro and Fairbairn, 1969). Given that after the penetration into the muscle fibers the larvae increase in size by more than 10 times in the first 20 days of development (Castro and Fairbairn, 1969), then a significant increase in the absolute glycogen content in them is expected. From the 14th day after the invasion, newborn Trichinella larvae started penetrating into the muscle fibers. This is the time of the active nutrition and growth of muscular Trichinella larvae in the host's myocytes (Castro and Fairbairn, 1969). It is possibly due to an increase in the size of muscular Trichinella larvae that the glycogen concentration in them is increasing. In the present study, a small amount of glycogen in the 14-day-old Trichinella larvae was found. On the 28th day after the invasion, the glycogen concentration in Trichinella larvae increased by 4.9 times, from $0.0028 \mu \mathrm{g} / \mathrm{larva}$ to $0.0136 \mu \mathrm{g} / \mathrm{larva}$.

Until one and a half months old, the larvae continue to actively accumulate glycogen. However, from the $45^{\text {th }}$ to $134^{\text {th }}$ days after infection, the intensity of storing the reserve substance is significantly reduced. Some researchers noted this phenomenon already after the 28th day of the invasion (Stewart, 1976; Wu et al., 2009). Pereverzeva (1966), using the histological method, registered the maximum glycogen content in the 6-month-old larvae. After completion of the organogenesis of muscle larvae and capsule formation, the supply of glycogen in invasive Trichinella larvae undergoes a sharp increase. The glycogen synthesis is likely to depend on different factors including the immune response of the host, the host species, the host gender and age, and nutrition conditions of the host. The viability of the larvae after digestion in gastric juice decreases by this time by $5 \%$, which is probably due to capsule calcification. However, this decrease can be considered insignificant- and does not affect the invasive capacity indicators.

In previous studies, other researchers determined the glycogen content in Trichinella using the less specific anthrone method. They established that the glycogen concentration in the viable larvae is about $0.0784 \mu \mathrm{g} / \mathrm{larva}$ (De Nollin and Van den Bossche, 1973). This value corresponds with the data obtained in the current study.

The fact that the total amount of glycogen contained in the larvae increases with growth and development, and then remains at the same level for a long time, indicates that this energetic compound is essential at a later stage when the larva turns into an adult Trichinella in the intestines of the host (Beckett and Boothroyd, 1962).

\section{Intestinal worms}

In the first hours of development in the intestines of the host, Trichinella spiralis worms have a very high energy expenditure associated with the invasion into intestinal mucosa, organogenesis, preparation for reproduction and the first molting process. Kozar (1973) observed that adult nematodes of Trichinella spiralis do not accumulate glycogen, but use its storage formed at the larval stage of development. Due to the low permeability of the cuticle at this stage, the reserve nutrient material enters the mid-intestine and breaks up there, apparently, into oligo- and monosaccharides. After 20 hours in the host's intestines, the cuticle that its permeability had increased 8 times by that time, is responsible for supplying nutritional substances and removing metabolites from the nematodes (Ferguson and Castro, 1973). Nevertheless, the Trichinella spiralis nematodes are highly dependent on the previously accumulated supplies of energy substances. Ferguson and Castro (1973) measured glycogen concentration in Trichinella spiralis localized in the intestine of mice. The glycogen content was $0.7 \%$ of dry weight of nematode 24 hours after infection. By the 4th day, this indicator increased to $1.3 \%$ (Kilgore et al., 1986). Kilgore (1986) detected a slightly higher value for the glycogen content $(4.9 \%)$ in 4-day-old nematodes. A relatively low amount of glycogen in adult worms disable them to survive in vitro for more than 12 hours in the absence of exogenous glucose (Kozar et al., 1966). Presence of Trichinella spiralis nematodes in the intestines of fasted mice for more than two days resulted in irreversible changes that led to the destruction and death of the helminths, and also after being kept in an isolated intestinal loop of a hamster, a delay in their developmental was observed (Timonov and Silakova, 1976).

\section{CONCLUSION}

The amount of glycogen stored at the muscle stage is extremely important in the first hours of the Trichinella spiralis development in the intestines of the host. The high energy requirements of Trichinella spiralis during the period when the nematodes cannot obtain enough nutrition, depend entirely on their glycogen storage. If the latter is insufficient, Trichinella spiralis nematodes lose their invasiveness.

\section{DECLARATIONS}

\section{Authors' contribution}

Evgenya A. Sidor contributed to data analysis and manuscript writing and performed the experimental works. Oleg N. Andreyanov was involved in the development of the methodology. Finally, all authors read and approved the final manuscript. 


\section{Competing interests}

The authors have declared no conflict of interest.

\section{Acknowledgments}

The authors express their gratitude to the vivarium staff All-Russian Scientific Research Institute of Fundamental and Applied Parasitology of Animals and Plants named after K.I. Skryabin - Branch of the Federal State Budget Scientific Institution «Federal Scientific Center - All-Russian Scientific Research Institute of Experimental Veterenari Medicina K.I. Skryabin and Y.R. Kovalenko the Russian Academy of Sciences», Moscow, Russia RAS.

\section{REFERENCES}

Andreyanov ON, Sidor EA and Timofeeva OG (2019). Patent No. 2681167 (13) C1 (RU) for the invention "Method for determining the quantity of glycogen in Trichinella larvae for monitoring the quality of neutralization of an invasive material". Bulletin No. 7 of 04.03.2019. Available at: https://www1.fips.ru/registers-doc-view/fips_servlet.

Beckett EB and Boothroyd B (1962). The histochemistry and electron microscopy of glycogen in the larva of Trichinella spiralis and its environment. Annals of Tropical Medicine \& Parasitology, 56 (3): 264-273. DOI: https://doi.org/10.1080/00034983.1962.11686119.

Bessonov AS (1975). Diagnosis of trichinosis. Vilnius: Mintis, p. 381.

Castro GA and Fairbairn D (1969). Carbohydrates and lipids in Trichinella spiralis larvae and their utilization in vitro. Journal of Parasitology, 55 (1): 51-58. Available at: https://www.ncbi.nlm.nih.gov/pubmed/5764047.

Danchenko EO and Chirkin AA (2010). A new methodological approach to determining glycogen concentration in tissues and some comments on the interpretation of the results. Forensic-Medical Examination, 3: 25-28. Available at: https://www.forensmed.ru/book.php?id=1277 .

De Nollin S and Van den Bossche H (1973). Biochemical effects of mebendazole on Trichinella spiralis larvae. Journal of Parasitology, 59 (6): 970-976. Available at: https://www.ncbi.nlm.nih.gov/pubmed/4760648.

European Convention for the Protection of Vertebrate Animals used for Experimental and other Scientific Purposes. ETS No.123. Strasbourg, 18/03/1986. Available at: http://conventions.coe.int /Treaty/EN/Treaties/PDF/123-Arev.pdf

Ferguson JD and Castro GA (1973). Metabolism of intestinal stages of Trichinella spiralis. American Journal of Physiology-Legacy Content, 225 (1): 85-89. Available at: https://www.ncbi.nlm.nih.gov/pubmed/4736619.

Kilgore MW and Stewart GL, Lou M (1986). Chemical composition of newborn larvae, muscle larvae and adult Trichinella spiralis. International Journal for Parasitology, 16 (5): 455-460. Available at: https://www.citeab.com/publication/2664690-2430908chemical-composition-of-newborn-larvae-muscle-larv /

Kozar Z, Zarzycki J, Seniuta R and Martynowicz T (1966). Histochemische untersuchungen über die darmphase der trichinellose bei weißen mäusen. Zeitschrift für Parasitenkunde, 27 (2): 106-126. Available at: https://link.springer.com/article/10.1007/BF00264131.

Montgomery JM, Augostini P and Stewart GL (2003). Glucose uptake and metabolism in the Trichinella spiralis nurse cell. International Journal for Parasitology, 33 (4): 401-412. Available at: https://www.ncbi.nlm.nih.gov/pubmed/12705933.

Okada H, Ikeda T, Kajita K, Mori I, Hanamoto T, Fujioka K, Yamauchi M, Usui T, Takahashi N, Kitada Y, et al. (2013). Effect of nematode Trichinella infection on glucose tolerance and status of macrophage in obese mice. Endocrine Journal, 60(11): 1241-9.

Patra G and Sarkar S (2014) Nurse cell biology of Trichinella spiralis. International Journal of Advancements in Research \& Technology, 3 (4): 133 - 138. Available at: http://www.ijoart.org/docs/Nurse-cell-Biology-of-Trichinella-spiralis.pdf .

Pereverzeva EV (1966). Histostructure changes of muscle tissue in experimental trichinosis in white mice. Scientists of the note of the Kursk State University, 23: 34-40.

Pereverzeva EV (1966). Histostructure changes of muscle tissue in experimental trichinosis in white mice. Scientists of the note of the Kursk State University, 23: 34-40.

Rudneva OV, Andreyanov ON and Sidor EA (2019). Changes in the level of glycogen and the invasive ability of the Trichinella nativa larvae stored in natural conditions. Scientia Parasitologyca, 20: 35 - 36. Available at: http://www.scientia.zooparaz.net/.

Sidor EA and Andreyanov ON (2019). Effect of positive temperatures on the glycogen content in Trichinella larvae. Collection of scientific articles adapted from the international scientific conference: theory and practice of parasitic disease control, 20: 585588. Available at: https://vniigis.ru/1_dlya_failov/Conferences\%20and\%20meetings/confenece-2019/\%D0\%9C\%20\%20\%D0\%A1\%20(49\%20\%D1\%81\%D1\%82\%D0\%B0\%D1\%82\%D0\%B5\%D0\%B9)/585-588.pdf .

Skvortsova FK and Uspensky AV (2016). Diagnostics of trichinosis in the early stages of larval development. Russian Journal of Parasitology, 1: 56-58. Available at: https://vniigis.elpub.ru/jour/article/view/237.

Skvortsova FK and Uspensky AV (2018). Determination role of Trichinella larvae at early stages of development in spread of trichinellosis. Rossiı̌skiı̌ Parazitologicheskiǐ Zhurnal, (3): 27-32.

Stewart GL (1976). Studies in biochemical pathology in trichinosis. II. Changes in liver and muscle glycogen and some blood chemical parameters in mice. Rice institute pamphlet-rice university studies., 62 (4): 211-224. Available at: https://scholarship.rice.edu/handle/1911/63250.

Timonov EV and Silakova LV (1976). Method of feeding Trichinella as a leading factor of adaptation to the mammalian organism. Parasitology, 6: 506-513.

Wu Z, Nagano I, Kajita K, Nishina M and Takahashi Y (2009). Hypoglycemia induced by Trichinella infection is due to the increase of glucose uptake in infected muscle cells. International Journal for Parasitology, 39 (4): 427-434. Available at: https://www.ncbi.nlm.nih.gov/pubmed/18838075. 